\title{
Crystal structure of dimethylglyoxime 2-picoloylhydrazone, $\mathrm{C}_{10} \mathrm{H}_{12} \mathrm{~N}_{4} \mathrm{O}_{2}$
}

\author{
H. Lin ${ }^{*, \mathrm{I}}$ and W.-S. Wu \\ I Jinhua Professional-Technical College, Jinhua, Zhejiang, 321017 P. R. China \\ II Huaqiao University, College of Materials, Quanzhou, Fujian, 362011 P. R. China
}

Received August 5, 2003, accepted and available on-line November 1, 2003; CCDC-No. 1267/1133

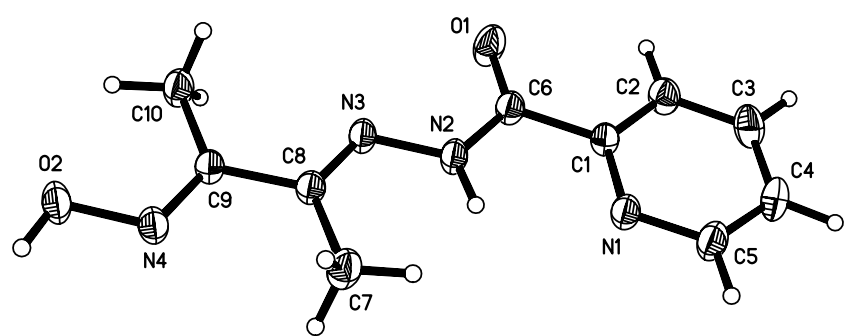

Abstract

$\mathrm{C}_{10} \mathrm{H}_{12} \mathrm{~N}_{4} \mathrm{O}_{2}$, monoclinic, $P 121 / c 1$ (No. 14), $a=5.7138(3) \AA$, $b=25.771(1) \AA, c=7.3379(4) \AA, \beta=100.385(2)^{\circ}, V=1062.8 \AA^{3}$, $Z=4, R_{\mathrm{gt}}(F)=0.051, w R_{\mathrm{ref}}\left(F^{2}\right)=0.170, T=293 \mathrm{~K}$.

\section{Source of material}

The title compound was synthesized by refluxing an equimolar mixture of 2-picoloylhydrazine and dimethylglyoxime in ethanol for $1 \mathrm{~h}$. Single crystals suitable for the X-ray structure analysis were obtained from ethanol.

\section{Discussion}

Hydrazides form an important class of organic compounds with many applications in organic synthesis and in industry. They are useful starting materials for the synthesis of biologically active heterocycles [1].

The title compound has essentially planar molecules (r.m.s. deviation from the least-squares mean plane is only $0.049 \AA$ ). The C8-N3 bond length of 1.283(2) $\AA$ is shorter than the C6-N2 one of $1.339(2) \AA$, indicating that $\mathrm{C} 8-\mathrm{N} 3$ is a double bond. A comparison of the picolonylhydrazide moiety's bond distances and angles in the title compound with propionylpicoloylhydrazide [2] show smaller differences. The molecules pack in a one-dimensional infinite chain along the $a$ axis because of the intermolecular $\mathrm{O} 2-\mathrm{H} \cdots \mathrm{O} 1^{a}$ hydrogen bonds $[d(\mathrm{O} 2 \cdots \mathrm{O} 1 \mathrm{a})=$ $2.689 \AA$ and $\angle \mathrm{O} 2-\mathrm{H} \cdots \mathrm{O} 1^{a}=153.4^{\circ}$ ] between the oximo-O atom in one molecule and the carbonyl-O atom in the orther molecule (symmetry code: $x+1,-y+1 / 2, z+1 / 2$ ).

Table 1. Data collection and handling.

\begin{tabular}{ll}
\hline Crystal: & colourless prism, \\
& size $0.23 \times 0.26 \times 0.80 \mathrm{~mm}$ \\
Wavelength: & Mo $K_{\alpha}$ radiation $(0.71069 \AA)$ \\
$\mu:$ & $1.00 \mathrm{~cm}^{-1}$ \\
Diffractometer, scan mode: & Rigaku R-AXIS RAPID, $\omega / \varphi$ \\
$2 \theta_{\text {max }}:$ & $54.96^{\circ}$ \\
$N(h k l)_{\text {measured }}, N(h k l)_{\text {unique }}:$ & 2424,2424 \\
Criterion for $I_{\text {obs }}, N(h k l)_{\mathrm{gt}}:$ & $I_{\text {obs }}>2 \sigma\left(I_{\text {obs }}\right), 1863$ \\
$N(\text { param })_{\text {refined }}$ & 146 \\
Programs: & SHELXS-97 [3], SHELXL-97 [4]
\end{tabular}

Table 2. Atomic coordinates and displacement parameters (in $\AA^{2}$ ).

\begin{tabular}{llllll}
\hline Atom & Site & $x$ & $y$ & $z$ & $U_{\text {iso }}$ \\
\hline $\mathrm{H}(2 \mathrm{O})$ & $4 e$ & 1.0133 & 0.1564 & 0.5589 & 0.062 \\
$\mathrm{H}(2 \mathrm{~N})$ & $4 e$ & 0.7307 & 0.3955 & 0.3814 & 0.049 \\
$\mathrm{H}(2)$ & $4 e$ & 0.1446 & 0.4911 & 0.1198 & 0.057 \\
$\mathrm{H}(3)$ & $4 e$ & 0.2472 & 0.5799 & 0.1179 & 0.068 \\
$\mathrm{H}(4)$ & $4 e$ & 0.6345 & 0.6047 & 0.2554 & 0.068 \\
$\mathrm{H}(5)$ & $4 e$ & 0.9018 & 0.5421 & 0.3911 & 0.063 \\
$\mathrm{H}(7 \mathrm{~A})$ & $4 e$ & 0.9650 & 0.3646 & 0.5056 & 0.080 \\
$\mathrm{H}(7 \mathrm{~B})$ & $4 e$ & 1.0045 & 0.3193 & 0.6504 & 0.080 \\
$\mathrm{H}(7 \mathrm{C})$ & $4 e$ & 1.1036 & 0.3147 & 0.4653 & 0.080 \\
$\mathrm{H}(10 \mathrm{~A})$ & $4 e$ & 0.5073 & 0.1840 & 0.3695 & 0.073 \\
$\mathrm{H}(10 \mathrm{~B})$ & $4 e$ & 0.3684 & 0.2333 & 0.4158 & 0.073 \\
$\mathrm{H}(10 \mathrm{C})$ & $4 e$ & 0.4515 & 0.2290 & 0.2241 & 0.073 \\
& & & & & \\
\hline
\end{tabular}

Table 3. Atomic coordinates and displacement parameters (in $\AA^{2}$ ).

\begin{tabular}{|c|c|c|c|c|c|c|c|c|c|c|}
\hline Atom & Site & $x$ & $y$ & $z$ & $U_{11}$ & $U_{22}$ & $U_{33}$ & $U_{12}$ & $U_{13}$ & $U_{23}$ \\
\hline $\mathrm{O}(1)$ & $4 e$ & $0.2129(3)$ & $0.39569(6)$ & $0.1815(2)$ & $0.0445(8)$ & $0.0396(7)$ & $0.096(1)$ & $-0.0083(6)$ & $-0.0181(7)$ & $0.0047(7)$ \\
\hline $\mathrm{O}(2)$ & $4 e$ & $0.8762(3)$ & $0.16875(5)$ & $0.5057(2)$ & $0.0550(8)$ & $0.0306(7)$ & $0.0670(9)$ & $0.0082(6)$ & $0.0025(6)$ & $0.0056(6)$ \\
\hline $\mathrm{N}(1)$ & $4 e$ & $0.6922(3)$ & $0.48113(6)$ & $0.3326(2)$ & $0.0404(8)$ & $0.0318(7)$ & $0.0530(9)$ & $-0.0013(6)$ & $-0.0013(6)$ & $-0.0013(6)$ \\
\hline $\mathrm{N}(2)$ & $4 e$ & $0.5909(3)$ & $0.38082(5)$ & $0.3317(2)$ & $0.0397(8)$ & $0.0272(7)$ & $0.0524(9)$ & $-0.0012(6)$ & $-0.0033(6)$ & $-0.0002(6)$ \\
\hline $\mathrm{N}(3)$ & $4 e$ & $0.5650(3)$ & $0.32793(5)$ & $0.3422(2)$ & $0.0403(8)$ & $0.0272(7)$ & $0.0456(8)$ & $-0.0006(5)$ & $0.0007(6)$ & $0.0000(6)$ \\
\hline $\mathrm{N}(4)$ & $4 e$ & $0.9104(3)$ & $0.22237(6)$ & $0.5103(2)$ & $0.0439(8)$ & $0.0322(7)$ & $0.0485(9)$ & $0.0036(6)$ & $0.0042(6)$ & $0.0027(6)$ \\
\hline$C(2)$ & $4 e$ & $0.3017(4)$ & $0.50259(7)$ & $0.1735(3)$ & $0.045(1)$ & $0.0400(9)$ & $0.053(1)$ & $0.0048(8)$ & $-0.0038(8)$ & $0.0041(8)$ \\
\hline
\end{tabular}

\footnotetext{
* Correspondence author (e-mail: jh_11@263.net)
} 
Table 3. Continued.

\begin{tabular}{|c|c|c|c|c|c|c|c|c|c|c|}
\hline Atom & Site & $x$ & $y$ & $z$ & $U_{11}$ & $U_{22}$ & $U_{33}$ & $U_{12}$ & $U_{13}$ & $U_{23}$ \\
\hline $\mathrm{C}(3)$ & $4 e$ & $0.3612(4)$ & $0.55454(8)$ & $0.1736(3)$ & $0.069(1)$ & $0.0363(9)$ & $0.061(1)$ & $0.0146(9)$ & $0.002(1)$ & $0.0072(9)$ \\
\hline $\mathrm{C}(4)$ & $4 e$ & $0.5871(4)$ & $0.56897(7)$ & $0.2536(3)$ & $0.075(1)$ & $0.0281(8)$ & $0.066(1)$ & $-0.0037(9)$ & $0.010(1)$ & $-0.0007(8)$ \\
\hline $\mathrm{C}(5)$ & $4 e$ & $0.7449(4)$ & $0.53154(8)$ & $0.3330(3)$ & $0.053(1)$ & $0.0359(9)$ & $0.065(1)$ & $-0.0074(8)$ & $0.0017(9)$ & $-0.0049(9)$ \\
\hline $\mathrm{C}(6)$ & $4 e$ & $0.4109(3)$ & $0.41094(7)$ & $0.2506(2)$ & $0.0394(9)$ & $0.0327(8)$ & $0.044(1)$ & $-0.0008(7)$ & $-0.0005(7)$ & $-0.0002(7)$ \\
\hline $\mathrm{C}(7)$ & $4 e$ & $0.9755(3)$ & $0.32766(8)$ & $0.5208(3)$ & $0.044(1)$ & $0.0396(9)$ & $0.069(1)$ & $-0.0049(8)$ & $-0.0108(9)$ & $0.0054(9)$ \\
\hline $\mathrm{C}(8)$ & $4 e$ & $0.7470(3)$ & $0.30332(6)$ & $0.4283(2)$ & $0.0383(8)$ & $0.0308(8)$ & $0.0404(9)$ & $-0.0006(6)$ & $0.0035(7)$ & $0.0003(6)$ \\
\hline $\mathrm{C}(10)$ & $4 e$ & $0.4917(3)$ & $0.22092(7)$ & $0.3537(3)$ & $0.045(1)$ & $0.0328(8)$ & $0.063(1)$ & $-0.0030(7)$ & $-0.0028(8)$ & $0.0010(8)$ \\
\hline
\end{tabular}

\section{References}

1. Wolter, M.; Klapars, A.; Buchwald, S. L.; Synthesis of $N$-aryl hydrazides by copper-catalyzed coupling of hydrazides with aryl iodides. Org. Lett. 3 (2001) 3803-3805.

2. Wu, W.-S.; Liu, S.-X.; Synthesis and Crystal structure of $N$-Propionylpicoloylhydrazide $\left(\mathrm{C}_{9} \mathrm{H}_{11} \mathrm{~N}_{3} \mathrm{O}_{2}\right)$. Chinese J. Struct. Chem. 20 (2001) 226-228.
3. Sheldrick, G. M.: Phase Annealing in SHELX-90: Direct Methods for Larger Structures. Acta Crystallogr. A46 (1990) 467-473.

4. Sheldrick, G. M.: SHELXL-97. Program for the refinement of crystal structures. University of Göttingen, Germany 1997. 